\title{
METHICILLIN-RESISTANT STAPHYLOCOCCUS AUREUS, EXTENDED SPECTRUM BETALACTAMASE AND METALLOBETALACTAMASE PRODUCTION AMONG ORGANISMS CAUSING SURGICAL SITE INFECTIONS AT A TERTIARY CARE HOSPITAL IN BANGALORE
}

\author{
Swati Jain'1, Anjana Gopi2, Faiza Samreen³, Madhulatha C. $K^{4}$ \\ ${ }_{13}^{\text {rd }}$ Year Junior Resident, Department of Microbiology, Kempegowda Institute of Medical Sciences and Research Hospital. \\ 2 Professor and HOD, Department of Microbiology, Kempegowda Institute of Medical Sciences and Research Hospital. \\ ${ }^{3} 3^{\text {rd }}$ Year Junior Resident, Department of Microbiology, Kempegowda Institute of Medical Sciences and Research Hospital. \\ ${ }^{4}$ Assistant Professor, Department of Microbiology, Kempegowda Institute of Medical Sciences and Research Hospital.
}

\begin{abstract}
BACKGROUND

Infection at or near surgical incisions within 30 days of an operative procedure, or within 1 year of an operation if a foreign body is implanted as part of the surgery is defined as a surgical site infection (SSI). They are the $2^{\text {nd }}$ most common cause of nosocomial infections. It has been estimated that SSI develops in at least $2 \%$ of hospitalised patients undergoing operative procedures. The incidence of Methicillin-Resistant Staphylococcus aureus (MRSA), extended spectrum betalactamase (ESBL) and metallobetalactamase (MBL) producing organisms have been steadily increasing over the past few years resulting in limitation of therapeutic options.
\end{abstract}

\section{MATERIALS AND METHODS}

It is a descriptive study conducted from December 2014 to June 2016 at a tertiary care hospital, Bangalore. Samples were collected aseptically from the wound using sterile cotton swabs. Isolates were identified by conventional methods and their antibiogram pattern was studied by Kirby Bauer's disc diffusion method. Staphylococcus aureus when isolated was studied for MRSA using oxacillin and cefoxitin disc diffusion test. Gram-negative isolates resistant to cephalosporins and carbapenems during antibiotic susceptibility testing were studied for ESBL production using ceftazidime and cefotaxime disc diffusion test and MBL production using imipenem-EDTA combined disc test respectively using standard CLSI guidelines. All the statistical analysis was done by chisquare test using SPSS software.

\section{RESULTS}

Of 100 clinically diagnosed cases, 68 samples yielded growth of various bacterial isolates. 47 out of 56 cases (83.9\%) from emergency surgeries and 21 out of 44 cases (47.7\%) from elective surgeries were infected. Staphylococcus aureus was the most common organism isolated (32.9\%) followed by Klebsiella pneumoniae (26.3\%). MRSA was observed in 24\% of Staphylococcus aureus isolates. ESBL production was observed in $23 \%$ of all Gram-negative isolates and MBL production was observed in $57 \%$ of all nil-fermenting Gram-negative isolates.

\section{CONCLUSION}

SSIs are menacing problem for healthcare providers. The rise of MRSA, ESBL and MBL producing organisms and continuous change in the resistance pattern has made SSI a therapeutic challenge. Management of SSI involves lot of skill and treatment should be judiciously based on risk stratification.

\section{KEYWORDS}

SSI, MRSA, ESBL and MBL.

HOW TO CITE THIS ARTICLE: Jain S, Gopi A, Samreen F, et al. Methicillin-resistant Staphylococcus aureus, extended spectrum betalactamase and metallobetalactamase production among organisms causing surgical site infections at a tertiary care hospital in Bangalore. J. Evolution Med. Dent. Sci. 2017;6(72):5123-5127, DOI: 10.14260/jemds/2017/1113

\section{BACKGROUND}

Surgical Site Infections [SSIs] have plagued surgeons since time immemorial.[1] Infection is encountered by all the surgeons; by nature of their craft, they invariably impair the first line of host defences, the cutaneous or the mucosal barrier. The entrance of microbes into the host tissues is the initial requirement for infection. [2]

Financial or Other, Competing Interest: None.

Submission 14-07-2017, Peer Review 25-08-2017,

Acceptance 31-08-2017, Published 07-09-2017.

Corresponding Author:

Dr. Anjana Gopi,

Aanchal, 19/33-1, $1^{\text {st }}$ Main, $2^{\text {nd }}$ Cross

Jayamahal Extension, Bangalore-46.

E-mail: dranjanagopi1989@gmail.com

DOI: $10.14260 /$ jemds $/ 2017 / 1113$

\section{(c) $(1)$}

SSIs are the second most common cause of nosocomial infections. It has been estimated that SSI develops in at least $2 \%$ of hospitalised patients undergoing operative procedures, although this is a likely underestimate because of incomplete post discharge data, other data indicate that SSIs develop following $3-20 \%$ of certain procedures. ${ }^{[3]}$

The Centres for Disease Control and Prevention (CDC) has proposed specific criteria for diagnosis of SSI. This splits SSIs into 3 groups- Superficial incisional, Deep incisional and Organ space infections depending on the site and the extent of infection.[4]

Sources of SSIs can include the patient's own normal flora or organisms present in the hospital environment. The common organisms encountered in post-operative wound infections are Staphylococcus aureus, Coagulase-Negative Staphylococci, Enterococci, Proteus, Pseudomonas, Escherichia coli and Klebsiella species. In case of wound 
infections following appendectomy or other lower bowel surgery, indigenous flora of the lower gastrointestinal tract are involved like Escherichia coli.[5]

Before the routine use of prophylactic antibiotics, infection rates in the four surgical classifications (Clean, clean-contaminated, contaminated and dirty wounds) were $1-2 \%$ or less for clean wounds, $6-9 \%$ for clean-contaminated wounds, $13-20 \%$ for contaminated wounds and about $40 \%$ for dirty wounds. Since the introduction of routine prophylactic antibiotic use, infection rates in the most contaminated groups have reduced drastically.[6]

The incidence of Methicillin-resistant Staphylococcus aureus (MRSA), extended spectrum betalactamases (ESBL) and metallo-beta-lactamases (MBL) producing organisms have been steadily increasing over the past few years resulting in limitation of therapeutic options. ${ }^{[7]}$

This study is undertaken to isolate and identify the aerobic bacteria from clinically diagnosed cases of surgical site infections, study their antibiogram pattern, detect MRSA among the Staphylococcus aureus isolates and detect ESBL and MBL production in Gram-negative bacterial isolates.

\section{MATERIALS AND METHODS}

The present study was carried out in the Department of Microbiology, Kempegowda Institute of Medical Sciences, Hospital and Research Centre, Bangalore over a period of one and half year (December 2014 to June 2016). A total of 100 clinically diagnosed cases of surgical site infections admitted in various surgical wards were taken for the study.

Samples were collected aseptically from the wound using sterile cotton swabs. Isolates were identified by conventional methods and their antibiogram pattern was studied by Kirby Bauer's disc diffusion method. Staphylococcus aureus when isolated was studied for MRSA using oxacillin and cefoxitin disc diffusion test. Gram-negative isolates resistant to cephalosporins and carbapenems during antibiotic susceptibility testing were studied for ESBL production using ceftazidime and cefotaxime disc diffusion test and MBL production using imipenem-EDTA combined disc test respectively using standard CLSI guidelines.

All the statistical analysis was done by Chi-square test using SPSS software.

\section{RESULTS}

A total of 100 clinically diagnosed cases of surgical site infections of all ages and both sexes, admitted in various surgical wards at KIMS Hospital, Bangalore, were included in the study.

Of 100 clinically diagnosed cases of SSI, maximum number of patients were in the age group of 21-30 years (45\%), followed by $31-40$ years $(18 \%)$ and $41-50$ years (18\%). Out of 100 cases studied, 38 (38\%) were male patients and $62(62 \%)$ were female patients with a male to female ratio of $0.6: 1$. (Figure 1 )

Of 100 clinically diagnosed cases of SSI, 68 cases belonged to class I wound and 32 cases to class II. SSIs in clean surgeries were $60.3 \%$ (41 of 68 ) and clean-contaminated surgeries were $84.3 \%$ (27 of 32 ). (Figure 2 ).

Of 100 clinically diagnosed cases, 44 cases belonged to elective surgery and 56 cases belonged to emergency surgery. SSIs in elective surgeries were $47.7 \%$ (21 of 44 ) and emergency surgeries were $84 \%$ (47 of 56). (Figure 3).
In our study, all cases of SSI were diagnosed between $2^{\text {nd }}$ and $4^{\text {th }}$ postoperative day.

Of 100 clinically diagnosed cases of SSI, 68 samples showed growth in culture and 32 samples yielded no growth.

The difference between positive and negative Gram staining for prediction of subsequent culture results was statistically significant $(\mathrm{p}<0.01)$ by Fisher exact test. (Table 1).

Out of 68 culture-positive cases, 60 (88.24\%) samples yielded a single organism and two organisms were grown in each of the remaining $8(11.76 \%)$ samples.

Out of 68 culture-positive cases, there were 76 isolates altogether. Staphylococcus aureus (32.9\%) was the most common organism isolated followed by Klebsiella pneumoniae (26.3\%). (Table 2).

Gram-positive organisms showed the following susceptibility pattern: Vancomycin (100\%), gentamycin (93.33\%), linezolid (90\%), cefepime and tetracycline (73.33\%), oxacillin (70\%), ciprofloxacin and cotrimoxazole (60\%), clindamycin (53.33\%), cefoperazone (50\%), amoxyclav (46.67\%) and erythromycin (43.33\%).

Gram-negative organisms showed the following susceptibility pattern: Levofloxacin (97.43\%), imipenem (84.6\%), amikacin and gentamycin (79.5\%), piperacillintazobactam (76.92\%), cefepime $(61.53 \%)$, ciprofloxacin (43.6\%), cefoperazone (35.9\%), amoxy-clav and cefuroxime (33.33\%), cotrimoxazole (30.76\%) and ampicillin (7.7\%).

Nil-fermenting organisms showed the following susceptibility pattern: Amikacin, levofloxacin and tobramycin $(100 \%)$, gentamycin, netilmicin and piperacillin-tazobactam (71.4\%), cefepime (57.1\%), imipenem and meropenem (42.9\%), ceftriaxone-tazobactam and cefuroxime $(14.3 \%)$ and amoxy-clav (0\%).

Out of 25 Staphylococcus aureus strains, 6 (24\%) were MRSA both by oxacillin and cefoxitin disc diffusion test.

Out of 39 Gram-negative isolates, 9 (23.1\%) were found to be ESBL producers by ceftazidime plus ceftazidimeclavulanic acid and cefotaxime plus cefotaxime-clavulanic acid disc diffusion test. 6 out of $14(42.86 \%)$ E. coli were found to be ESBL producers and 3 out of 20 (15\%) K. pneumoniae were found to be ESBL producers.

Out of 7 nil-fermenting Gram-negative isolates, 4 (57.14\%) were found to be MBL producers by imipenemEDTA combined disc test. 2 out of $4(50 \%)$ A. baumannii were found to be MBL producers and 2 out of $3(66.67 \%)$ P. aeruginosa were found to be MBL producers.

\begin{tabular}{|c|c|c|c|c|}
\hline \multicolumn{2}{|c|}{ Gram Stain } & $\begin{array}{l}\text { Culture } \\
\text { Positive }\end{array}$ & $\begin{array}{c}\text { Culture } \\
\text { Negative }\end{array}$ & Total \\
\hline \multirow{3}{*}{ Positive } & PC \& GPC & 21 & 03 & \multirow{3}{*}{70} \\
\hline & PC \& GNB & 38 & 01 & \\
\hline & PC, GPC \& GNB & 07 & 00 & \\
\hline \multirow{2}{*}{ Negative } & Few PC, No org & 02 & 03 & \multirow{2}{*}{30} \\
\hline & No PC, No org & 00 & 25 & \\
\hline \multicolumn{2}{|r|}{ Total } & 68 & 32 & 100 \\
\hline
\end{tabular}

PC- Pus cells, GPC- Gram-positive cocci, GNB- Gramnegative bacilli, org- organism.

\begin{tabular}{|c|c|c|}
\hline Organisms & No. of Isolates & (\%) \\
\hline Staphylococcus aureus & 25 & 32.9 \\
\hline
\end{tabular}




\begin{tabular}{|c|c|c|}
\hline Klebsiella pneumoniae & 20 & 26.3 \\
\hline Escherichia coli & 14 & 18.42 \\
\hline Acinetobacter baumannii & 04 & 5.26 \\
\hline Enterobacter cloacae & 04 & 5.26 \\
\hline Enterococcus faecalis & 03 & 3.95 \\
\hline Pseudomonas aeruginosa & 03 & 3.95 \\
\hline Staphylococcus epidermidis & 02 & 2.63 \\
\hline Proteus mirabilis & 01 & 1.32 \\
\hline Total & $\mathbf{7 6}$ & $\mathbf{1 0 0}$ \\
\hline
\end{tabular}

Table 2. Distribution of Culture-positive Cases According to Spectrum of Bacterial Isolates

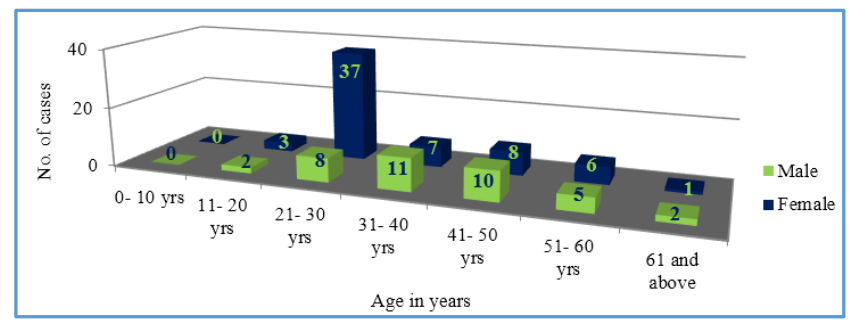

Figure 1. Age and Gender Distribution of Patients with SSI

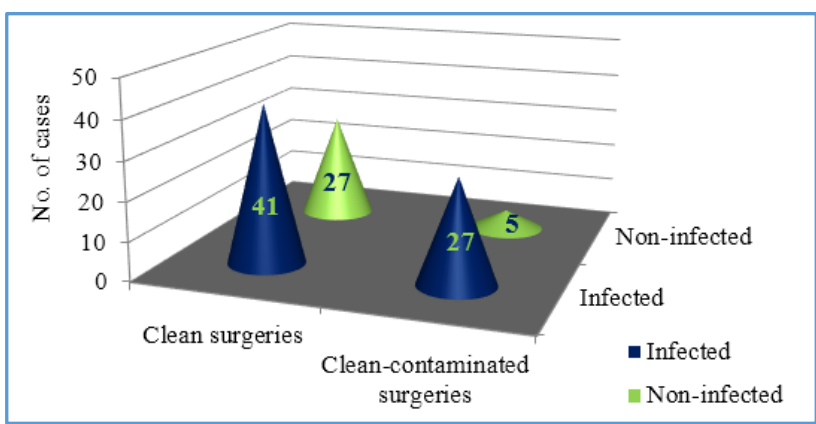

Figure 2. Comparison of SSI rates According to Class of Surgery

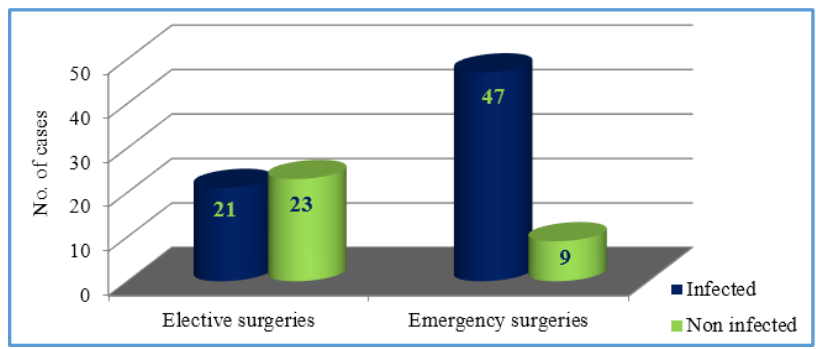

Figure 3: Comparison of SSI Rates According to Nature of Surgery

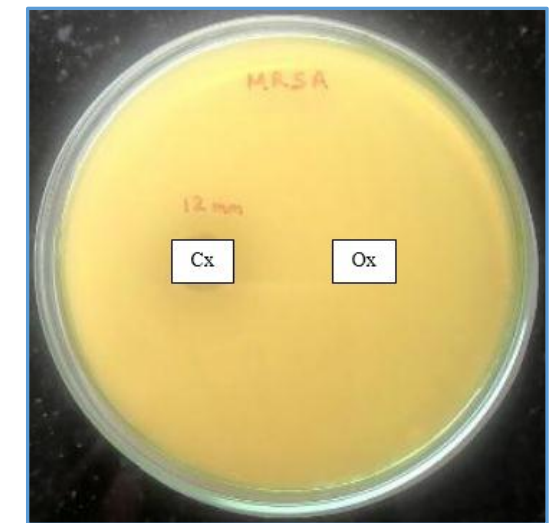

Picture 1: MRSA-Cefoxitin and Oxacillin Disc Diffusion Test

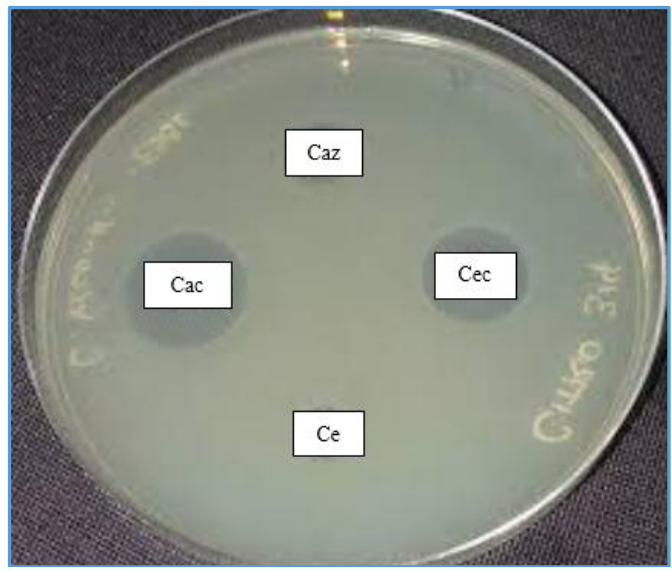

Picture 2: ESBL-Ceftazidime plus Ceftazidime/Clavulanic Acid and Cefotaxime plus Cefotaxime Clavulanic Acid Disc Diffusion Test

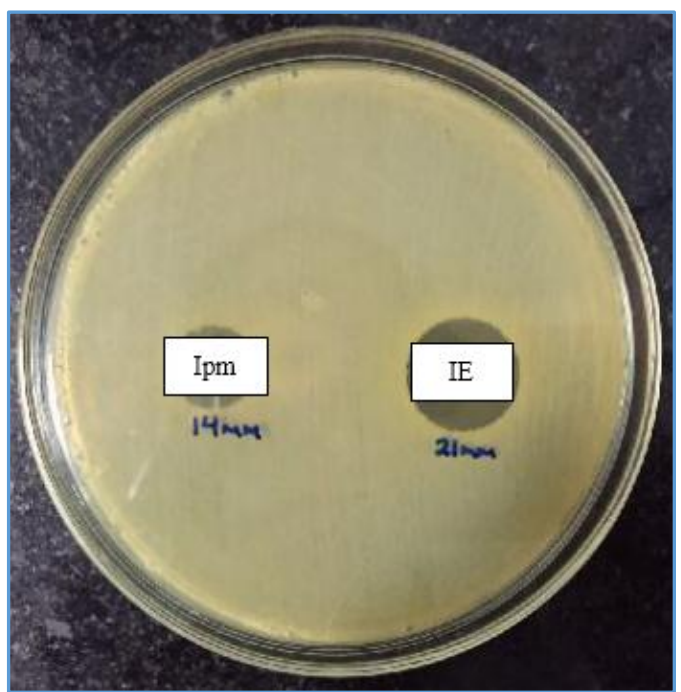

Picture 3: MBL-Imipenem-EDTA Combined Disc Test

\section{DISCUSSION}

Wound infection as a complication of both surgery and trauma has always been a challenge to surgeons. The management of infection is a complex and important aspect of wound care. The value of Microbiology in prevention and treatment of wound infection cannot be overemphasised, as proactive measures are made possible with knowledge of the prevailing organisms and their antibiogram. ${ }^{[8]}$

While the global estimates of SSIs have varied from 2.5$7 \%,{ }^{[9,10]}$ studies in India have consistently shown higher rates ranging from $23-38 \% \cdot{ }^{[11,12]}$ In our study, out of 100 clinically suspected cases of SSI, 68 were infected (infection rate of $68 \%$ ). Giacometti A et al in 2000 reported an overall SSI rate of $90.8 \%{ }^{[13]}$ Similar finding was noted by Divya P in 2016 (SSI rate of 92.9\%) in Bellary, Karnataka.[14] The variability in estimates is consistent with the differences in the characteristics of the hospital populations, the underlying diseases, differences in clinical procedures, the extent of infection control measures, and in addition the hospital environment.

The endogenous contamination remains the main risk factor for the development of surgical infection because of the enormous dose of the organisms available from the bowel and other hollow visceral organs. In our study, SSI rate for clean surgeries was $60.3 \%$, which increased to $84.3 \%$ in 
clean-contaminated, which was significant statistically $(p<0.05)$ and was in accordance with other studies. Lilani et al in 2005 noted a SSI rate of $3.03 \%$ in clean surgeries and $22.4 \%$ in clean-contaminated..15] Kamat et al in 2008 observed an infection rate of $5.4 \%$ for clean and $35.5 \%$ for clean-contaminated in a descriptive study of SSIs in a teaching hospital in Goa.[12]

Higher incidence of wound infections was seen in emergency operations (84\%) in the present study than the elective operations $(47.7 \%)$ which was significant statistically $(\mathrm{p}<0.01)$. Kamat et al $(2008)$ also observed increased incidence of SSI in emergency cases. ${ }^{[12]}$ Higher infection rate in patients who underwent emergency surgery could be due to combination of various factors: patient's condition is generally low, inadequate preparation, operations performed are usually on potentially contaminated or contaminated sites, i.e. intestinal obstruction, strangulated hernia, etc.

In the present study, 76 isolates were obtained from 68 culture-positive cases of SSI. Most common organism isolated was Staphylococcus aureus (32.9\%), followed by Klebsiella pneumoniae (26.3\%) and Escherichia coli (18.42\%). Other organisms isolated were Acinetobacter baumannii and Enterobacter cloacae (5.26\% each), Enterococcus faecalis and Pseudomonas aeruginosa $(3.95 \%$ each $)$, Staphylococcus epidermidis (2.63\%) and Proteus mirabilis (1.32\%). Predominance of Staphylococcus aureus in SSI in present study is consistent with reports from other studies like Lilani et al (2005).[15] Among the Gram-negative bacilli, Klebsiella pneumoniae (43.48\%) was the most commonly isolated organism.

In the present study, among 25 isolates of Staphylococcus aureus, 6 strains (24\%) were methicillin resistant both by cefoxitin and oxacillin disc diffusion tests. According to Anand $\mathrm{KB}$, out of 50 strains of Staphylococcus aureus, 32 were MRSA by cefoxitin disc diffusion test and 28 were resistant by oxacillin disc diffusion test.[16]

In the present study, $42.86 \%$ Escherichia coli and $15 \%$ of Klebsiella pneumoniae isolates were found to be ESBL producers. This correlates with the study done by Kumar MS in which $63.7 \%$ of Escherichia coli and 14\% of Klebsiella pneumoniae isolates were found to be ESBL producers.[17]

In our study, $66.67 \%$ of Pseudomonas aeruginosa and $50 \%$ of Acinetobacter baumannii were found to be MBL producers. The percentage of MBL production in Pseudomonas aeruginosa is similar to studies conducted by Manoharan et al (42.6\%) and Saderi $\mathrm{H}$ et al $(39.06 \%) .[18,19]$

\section{CONCLUSION}

This study has given us the knowledge about SSIs and their incidence in our hospital, it also emphasised on the fact that SSIs are more common in clean-contaminated than in clean surgeries. It also showed that there was increased rate of SSIs in patients who underwent emergency surgeries.

It has given us the knowledge of the most common organisms causing surgical site infections and their antibiotic susceptibility pattern. Staphylococcus aureus was the commonest organism isolated, however, $60.5 \%$ of the isolates were Gram-negative bacilli with Klebsiella pneumoniae being the predominant isolate.
The Gram-positive organisms were most susceptible to vancomycin followed by gentamicin and linezolid. The Gramnegative organisms were most susceptible to levofloxacin followed by imipenem, amikacin and gentamicin.

Methicillin resistance is a common problem in Staphylococcus aureus which can be routinely detected by oxacillin and cefoxitin disc diffusion test. The MRSA strains in our study were most susceptible to vancomycin, gentamicin and linezolid.

Microorganisms like Klebsiella pneumoniae and Escherichia coli have the ability to produce Extended spectrum betalactamase resulting in limitation of therapeutic options which can be routinely detected by ceftazidime plus ceftazidime-clavulanic acid and cefotaxime plus cefotaximeclavulanic acid disc diffusion test. The ESBL strains in our study were most susceptible to levofloxacin followed by imipenem and gentamicin.

The presence of metallobetalactamases producing Pseudomonas aeruginosa and Acinetobacter baumannii is being increasingly reported and was detected in our study by imipenem-EDTA combined disc test. The MBL strains in our study were most susceptible to levofloxacin, amikacin and tobramycin.

Each hospital has its own unique bacterial flora to which patients are at risk for acquiring infection during hospitalisation. In such situations, microorganisms exhibit unique patterns of antimicrobial activity during a certain period of time. Only when such epidemiological data are available can the surgeon employ a logical approach towards SSI control. Resistance to antimicrobials also has become a serious problem necessitating in depth study of SSI to prevent future complications in operated cases.

Understanding the factors leading to surgical site infection will help in rationalising the use of antimicrobials as it now appears that the wheel has rotated a full circle and fear is rightly being expressed that we will soon have the same conditions of wound infection as before Lister's era, if not judiciously used.

The presence of MRSA, ESBL and MBL in our study calls for intensive infection control practices, and continuous antibiotic surveillance and an effective antibiotic policy are required to address the problem.

Molecular studies are necessary to evaluate the various MRSA, ESBL and MBL types.

\section{REFERENCES}

[1] Jain K, Chavan NS, Jain SM. Bacteriological profile of post-surgical wound infection along with special reference to MRSA in central India, Indore. Int J Intg Med Sci 2014;1(1):9-13.

[2] Howard RJ. Surgical infections. In principles of surgery. vol. 1. $7^{\text {th }}$ edn. Schwartz, McGraw Hill 123-52.

[3] Klevens RM, Edwards JR, Richards CL, et al. Estimating health care-associated infections and deaths in U.S. hospitals, 2002. Public Health Rep 2007;122(2):1606.

[4] Mahmoud NK, Merril TD. Surgical complications. In: Townsend MC, Beauchamp RD, Evers BM, et al. (eds). Sabiston textbook of surgery. $19^{\text {th }}$ edn. New Delhi: Elsevier, 2008;1:328-70. 
[5] Forbes BA, Sahm DF, Weissfeld AS. Skin, soft tissue and wound infections. In: Bailey and Scott's diagnostic microbiology. $11^{\text {th }}$ edn. St louis: Mosby 2002:978-9.

[6] Cruse PJ, Foord R. The epidemiology of wound infection. A ten year prospective study of 62,939 wounds. Surg Clin North Am 1980;60(1):27-40.

[7] Aggarwal R, Goel N, Choudhary U, et al. Prevalence of MRSA as uropathogen in a teaching tertiary care hospital of north India. Int J Pharm Med and Bio Sc 2013;2(2):18-22.

[8] Thanni LOA, Osinupebi OA, Deji-Agboola M. Prevalence of bacterial pathogens in infected wounds in a tertiary hospital, 1995-2001: any change in trend? J Natl Med Assoc 2003;95(12):1189-95.

[9] Olson M, O'Connor M, Schwartz ML. Surgical wound infections. A 5-year prospective study of 20,193 wounds at the Minneapolis VA medical center. Ann Surg 1984;199(3):253-9.

[10] Shrestha S, Wenju P, Shrestha R, et al. Incidence and risk factors of surgical site infections in Kathmandu university hospital, Kavre, Nepal. Kathmandu Univ Med J 2016;14(54):107-11.

[11] Ganguly PS, Khan Y, Malik A. Nosocomial infections and hospital procedures. Indian J Commun Med 2000;25(1):39-43.

[12] Kamat US, Fereirra AM, Kulkarni MS, et al. A prospective study of surgical site infections in a teaching hospital in Goa. Indian J Surg 2008;70(3):120-4.
[13] Giacometti A, Cirioni 0, Schimizzi AM, et al. Epidemiology and microbiology of surgical wound infections. J Clin Microbiol 2000;38(2):918-22.

[14] Divya P. Prevalence and susceptibility pattern of staphylococcus aureus in post-operative surgical wound infections at tertiary care hospital. IJHSR 2015;5(7):129-32.

[15] Lilani SP, Jangale N, Chowdhary A, et al. Surgical site infection in clean-contaminated cases. Indian J Med Microbiol 2005;23(4):249-52.

[16] Anand KB, Agrawal P, Kumar S, et al. Comparison of cefoxitin disc diffusion test, oxacillin screen agar, and PCR for mecA gene for detection of MRSA. Indian J Med Microbiol 2009;27(1):27-9.

[17] Kumar MS, Lakshmi V, Rajagopalan R. Occurrence of extended spectrum beta-lactamases among Enterobacteriaceae spp. isolated at a tertiary care institute. Indian J Med Microbiol 2006;24(3):208-11.

[18] Manoharan A, Chatterjee S, Mathai D. Detection and characterization of metallo beta lactamases producing Pseudomonas aeruginosa. Ind J Med Microbiol 2010;28(3):241-4.

[19] Goel V, Hogade SA, Karadesai SG. Prevalence of extended-spectrum beta-lactamases, AmpC betalactamase and metallo-beta-lactamase producing Pseudomonas aeruginosa and Acinetobacter baumannii in an intensive care unit in a tertiary care hospital. J Sci Soc 2013;40(1):28-31. 